\title{
Usefulness and Safety of a Guide Catheter Extension System for the Percutaneous Treatment of Complex Coronary Lesions by a Transradial Approach
}

\author{
Sergio García-Blas Julio Núñez Luis Mainar Gema Miñana Clara Bonanad \\ Paolo Racugno Juan Carlos Rodríguez Patricia Moyano Juan Sanchis
}

Department of Cardiology, Hospital Clínico Universitario de Valencia, Valencia, Spain

\section{Key Words}

Coronary artery disease $\cdot$ Percutaneous coronary

intervention · Transradial approach · GuideLiner ${ }^{\circledR}$ catheter

\begin{abstract}
Objective: The aim of this study was to describe our initial experience with the GuideLiner ${ }^{\circledR}$ catheter (Vascular Solutions Inc.) in the transradial treatment of complex lesions. Materials and Methods: The clinical, angiographic and procedural data of percutaneous coronary interventions where GuideLiner was used during 2013 were collected. The transradial approach was used in all cases. The indication for its use, efficacy and periprocedural complications were determined. Sixteen consecutive procedures (in 15 patients; 12 males and 3 females) were evaluated. The indication for the use of GuideLiner was a difficulty to advance and properly position a stent through a tortuous and/or calcified artery despite using high-support guide catheters or other useful techniques. Results: Of the 16 angiographic procedures, 14 (87.5\%) were successful (stent deployment in 13 cases and a drug-eluting balloon in 1 case). Unsuccessful cases were a chronic total occlusion and a diffusely diseased left anterior descendant artery. A type B dissection of a proximal left cir-
\end{abstract}

cumflex artery was the only periprocedural complication. Conclusion: Use of the GuideLiner was an effective and safe technique for the percutaneous treatment of complex coronary lesions in which the adequate progress of angioplasty devices had failed. GuideLiner was particularly helpful when using the transradial approach. Only one minor complication was recorded.

(c) 2014 S. Karger AG, Basel

\section{Introduction}

The percutaneous treatment of complex coronary lesions is still a challenging problem, especially when using the transradial approach. The presence of calcification, marked tortuosity or treatment of total chronic occlusions and the difficulty in the use of angioplasty balloons and stents lead to a considerable failure of stent deployment (approximately 3\%) or to the development of other complications $[1,2]$.

Currently, the radial approach is the most widely used in our setting due to its efficiency and low complication rate [3-5], in spite of the development of useful femoral closure devices [6]. Nevertheless, the strength of support

\begin{tabular}{|c|c|}
\hline KARGER 125 & $\begin{array}{l}\text { (c) 2014 S. Karger AG, Basel } \\
1011-7571 / 14 / 0242-0171 \$ 39.50 / 0\end{array}$ \\
\hline $\begin{array}{l}\text { E-Mail karger@karger.com } \\
\text { www.karger.com/mpp }\end{array}$ & $\begin{array}{l}\text { This is an Open Access article licensed under the terms of the } \\
\text { Creative Commons Attribution-NonCommercial 3.0 Un- } \\
\text { ported license (CC BY-NC) (www.karger.com/OA-license), } \\
\text { applicable to the online version of the article only. Distribu- } \\
\text { tion permitted for non-commercial purposes only. }\end{array}$ \\
\hline
\end{tabular}


offered by the radial approach is significantly lower than that for femoral access, which implies an extra difficulty in the percutaneous treatment of complex coronary lesions [7]. Patients with this type of lesion have not only an increased risk of vascular complications but also a greater comorbidity burden [8]. Theoretically, such patients are a group that may particularly benefit from the radial approach from a security point of view. Hence, new percutaneous coronary strategies maximizing the efficacy, while minimizing the risk of complications, are needed.

There are several techniques, such as the use of guide catheters with high passive back-up against the opposite aortic wall, active intubation of the guide, stiffer wires, anchoring or a 'buddy wire' [9-12], which can increase the strength of backup and support in complex cases. Among these techniques are guide catheter extension devices like the GuideLiner ${ }^{\circledR}$ catheter (Vascular Solutions Inc.) that permits deep intubation and provides greater support and coaxiality [13]. The aim of the present study was to describe the efficacy and safety of the use of this type of device in the treatment of complex lesions with the radial approach based on the initial experience at our center.

\section{Subjects and Methods}

All consecutive cases (15 in total; 12 males and 3 females) of percutaneous coronary intervention (PCI) by a radial approach where the GuideLiner was used were prospectively included from January 1 to December 31, 2013. A total of 16 procedures were performed in the 15 patients. Stable angina was the indication for catheterization in only 4 cases; the remaining procedures were performed in acute coronary syndromes: 1 primary angioplasty for ST elevation myocardial infarction, 8 for non-ST elevation myocardial infarction, and the last 3 that were planned deferred revascularization at a nonculprit lesion diagnosed during primary angioplasty (table 1). The left anterior descending (LAD) artery was treated in 7 cases, the right coronary artery was treated in 5 cases, and the circumflex $(\mathrm{Cx})$ artery was treated in 4 cases. In all but 1 case, the affected segment was medium or distal.

High-support catheters were chosen (extra-backup XB, Amplatz left, Amplatz right, sheathless SPB3), except in 2 cases where Judkins R4 was used. The indication for using GuideLiner was the difficulty to advance and properly position a stent through a tortuous or calcified artery despite using other useful techniques in this context (deep intubation with the guiding catheter, a stiff guidewire, predilatation with noncompliant balloons or a 1:1 balloon: vessel ratio, 'buddy wire' or 'anchoring'.

The indication for catheterization and revascularization, periprocedural treatment and all technical aspects were left to the discretion of the operator, who followed the usual clinical practice. The radial approach was used in all cases, with a 6-Fr sheath except for one 6.5-Fr sheathless guide catheter (ASAHI; Vascular Perspectives Ltd). Clinical information (medical history, current clinical presentation and coronary angiography indication), angio-
Table 1. Patient baseline characteristics

\begin{tabular}{ll}
\hline Patients & 15 \\
Age, years & $72 \pm 8.3$ \\
Gender & \\
$\quad$ Male & $12(80)$ \\
$\quad$ Female & $3(20)$ \\
DM & $9(60)$ \\
Previous CAD & $8(53.3)$ \\
Previous PCI & $6(40)$ \\
Coronary angiography indication $(\mathrm{n}=16)$ & \\
$\quad$ Stable CAD & $4(25)$ \\
$\quad$ STEMI & $1(6.25)$ \\
$\quad$ Non-STEMI & $8(50)$ \\
$\quad$ Deferred & $3(18.75)$ \\
\hline
\end{tabular}

Data are presented as mean \pm SD or $\mathrm{n}(\%)$. DM $=$ Diabetes mellitus; $\mathrm{CAD}=$ coronary artery disease STEMI = ST segment elevation myocardial infarction; deferred = PCI on nonculprit coronary lesion diagnosed during a previous procedure.

graphic data (number of diseased vessels, type of lesion, presence of calcification or tortuosity), procedural data (type of guiding catheter, wire and predilatation) and the final result (angiographic success and complications) were recorded.

The GuideLiner V2 catheter extension device (Vascular Solutions Inc.) was used in all cases. This catheter consisted of a monorail system, which extends to the distal end of the guide catheter ('mother-child' fashion), with a length of $25 \mathrm{~cm}$, a thickness $1 \mathrm{Fr}$ less than the guide catheter and a design that minimizes trauma on the artery wall. The monorail continues proximally with a thin hypotube. The technique began with engaging the guiding catheter (mother) and positioning the guidewire. Next, the GuideLiner was inserted into the guide catheter through the hemostatic valve and advanced until it reached the coronary artery. This technique permitted deep intubation into the artery, providing great coaxiality and enhanced support.

Effectiveness was considered to be reached when the GuideLiner could be correctly used and adequately placed in the selected coronary artery. Efficacy of the procedure was defined as the achievement of optimal angiographic outcome with no significant residual stenosis and a TIMI 3 flow grade. For safety assessment, all clinical periprocedural complications were collected, as were major clinical events (death, myocardial infarction or repeated revascularization) at the 30 -day follow-up.

\section{Results}

The procedural characteristics are summarized in table 2. In all cases, it was possible to properly use the GuideLiner, obtaining a deep and selective intubation of the target artery. Of the 16 procedures, in 13 (81\%) it was 
Table 2. Summary of procedural characteristics

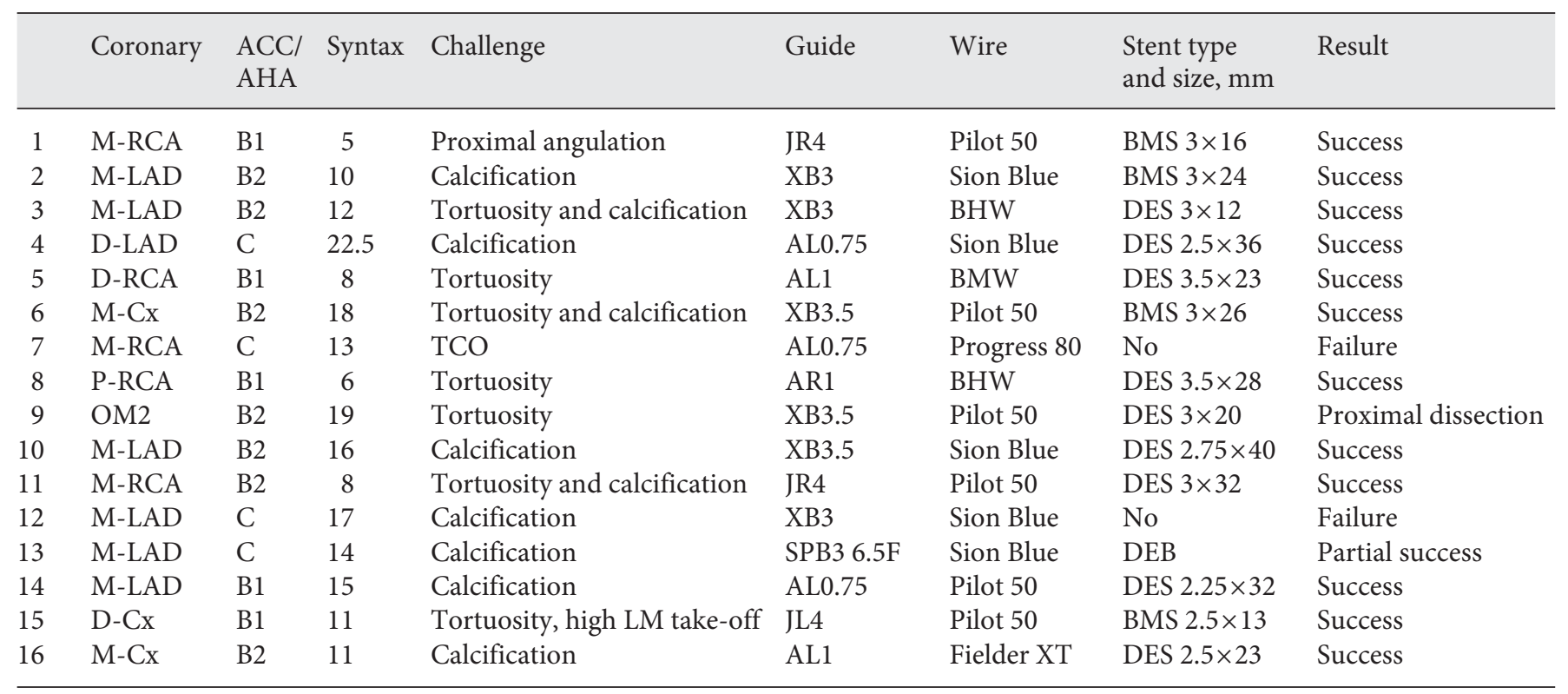

$\mathrm{P}=$ Proximal; $\mathrm{M}=$ mid; $\mathrm{D}=$ distal; $\mathrm{RCA}=$ right coronary artery; $\mathrm{OM} 2$ = second obtuse marginal; $\mathrm{TCO}=$ total coronary occlusion; $\mathrm{LM}=$ left main; JR = Judkins right; $\mathrm{XB}$ = extra back-up; $\mathrm{AL}=$ Amplatz left; $\mathrm{AR}=$ Amplatz right; $\mathrm{SPB}=$ sheathless power back-up; JL = Judkins left; $\mathrm{BHW}=$ balanced heavy weight; DES = drug-eluting stent; BMS = bare metal stent; DEB = drug-eluting balloon.

possible to position and deploy a stent, obtaining an optimal angiographic result (fig. 1a-d). The 2 failed cases involved a highly calcified mid-LAD lesion with a pronounced angulation and a chronic total occlusion in which GuideLiner use allowed only a partial advance of the balloon (insufficient to treat the entire length of the lesion). The remaining case was a mid-distal LAD highly calcified lesion in a vessel with diffuse disease in which a stent could not be deployed. Nevertheless, the use of GuideLiner enabled the advancement of a drug-eluting balloon. Therefore, the angiographic result was considered optimal in $14(87.5 \%)$ of the 16 cases.

Regarding the safety of its use, intubation with the GuideLiner was well tolerated, without significant pressure damping or ischemic symptoms. In 1 case, a type B dissection in the proximal Cx segment was produced, which was successfully sealed with a stent. It is noteworthy that in the aforementioned segment there was a nonsignificant atherosclerotic plaque which probably favored the occurrence of this complication (fig. 2). No other complications were detected during the hospitalization or 30-day follow-up of these patients.

\section{Discussion}

The results of this study show that the GuideLiner was a useful tool for approaching challenging coronary lesions using radial access. Guide extension catheter devices allowed a selective deep intubation of the coronary artery while it improved coaxiality and support, thus facilitating the treatment of complex lesions. Our results confirmed the previous report of the Twente GuideLiner registry that involved 65 patients in whom the device was shown to be effective for percutaneous treatment of complex lesions [14]. The GuideLiner, with its monorail system, facilitates clinical application, which is easier than with first coaxial devices (such as Heartrail Terumo ${ }^{\circledR}[2]$ ). Currently, there are also other commercially available monorail guide extender catheters, such as Guidezilla ${ }^{\mathrm{TM}}$ (Boston Scientific), Kiwami (Terumo, Tokyo, Japan) and Cokatte (Asahi Intecc). However, the latter two are not commercially available in the USA or Europe.

The most common indication for GuideLiner use is the difficulty or inability to place a stent or balloon in a complex coronary lesion. This problem occurs mainly in complex calcified lesions or tortuous arteries, as reflected in the type of lesions treated in our series. In the Twente 

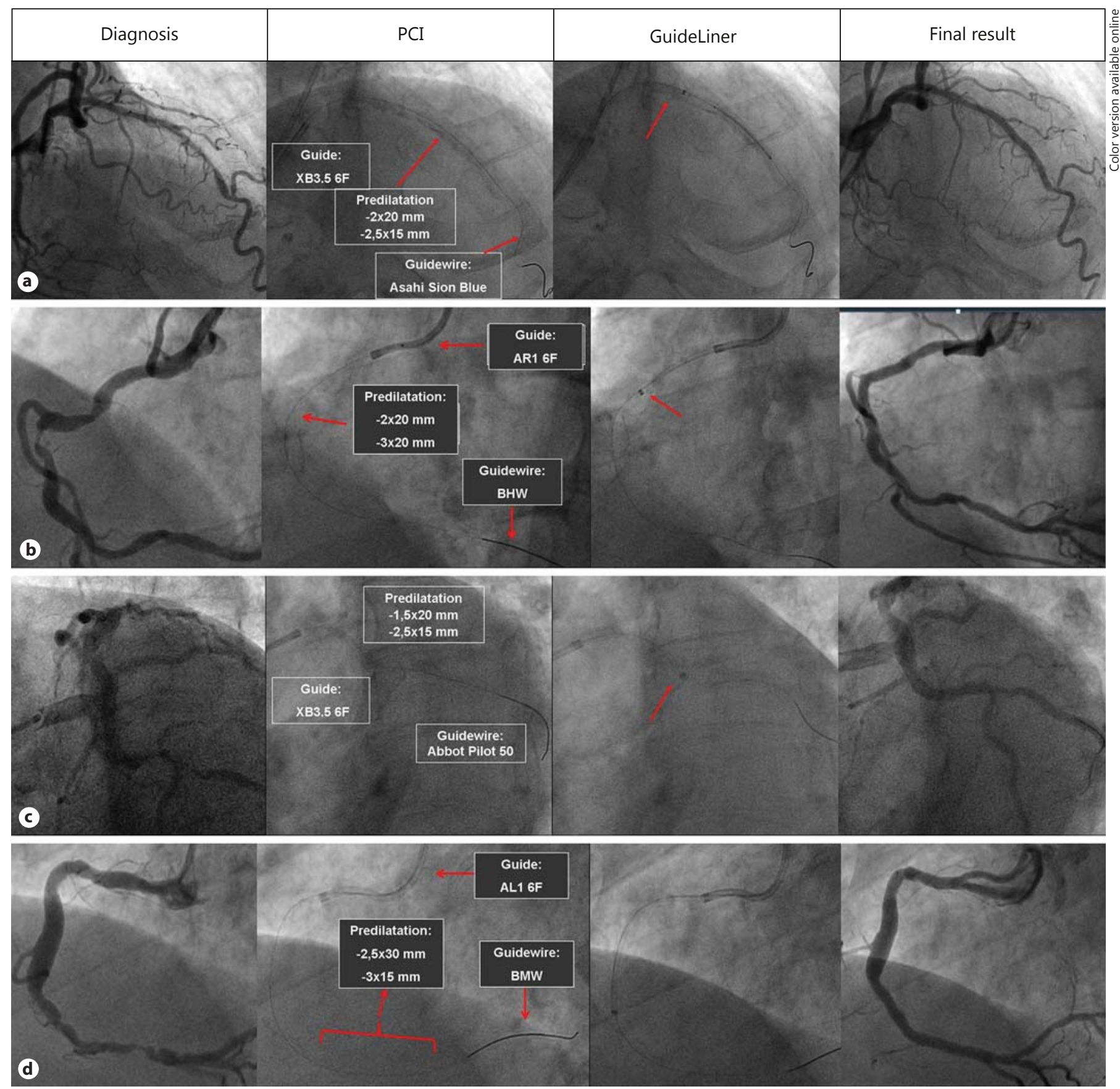

Fig. 1. Successful treatment of coronary lesions with GuideLiner. a Mid-LAD artery. b Mid-right coronary artery. c Mid-Cx artery. $\mathbf{d}$ Distal right coronary artery.

registry, $97 \%$ of the lesions were type $\mathrm{B} 2$ or $\mathrm{C}$ in the American Heart Association/American College of Cardiology (AHA/ACC) classification [14]. Both proximal vessel angulation and lesion angle have been identified as independent predictors for GuideLiner use [15]. The growing complexity of coronary lesions treated percutaneously makes this type of guide catheter extension a useful tool for an increasing number of cases in daily clinical practice. Our data showed that this technique is mainly applicable to mid or distal lesions in arteries (in 

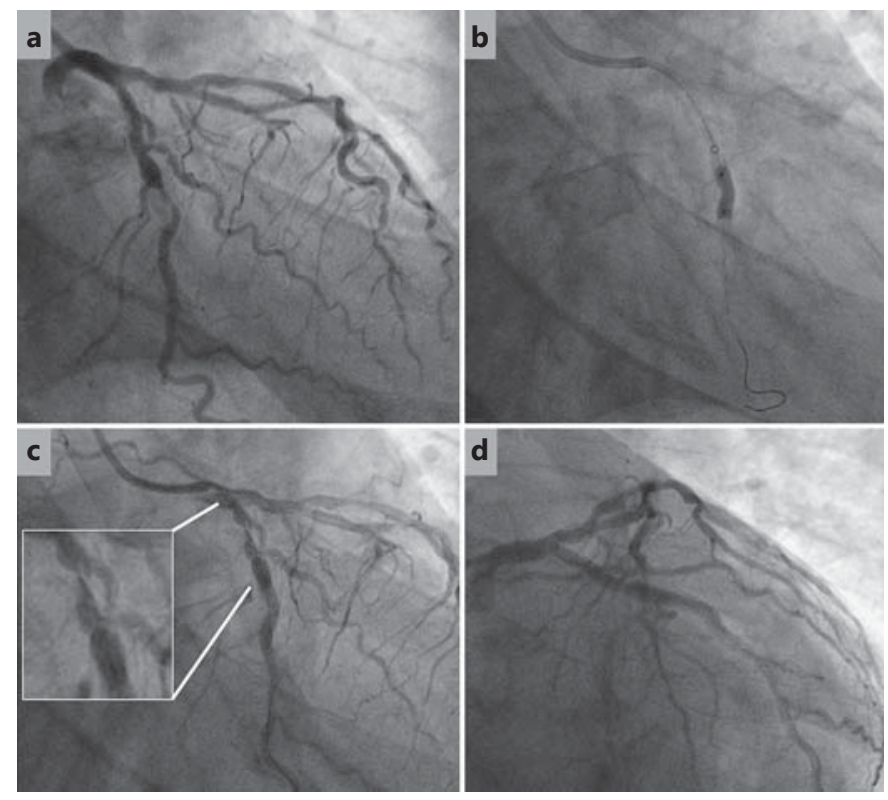

Fig. 2. Proximal Cx artery dissection after GuideLiner use. a Basal angiography of mid-Cx severe stenosis. b Use of GuideLiner to postdilate a mid-Cx stent. c Proximal Cx dissection due to GuideLiner. $\mathbf{d}$ Final result after placing a stent and sealing the dissection.

15 of 16 cases) with relatively preserved proximal segments that can safely accommodate the catheter extension. Other indications are less common, but can be equally useful, such as the engagement of the anomalous origin of a coronary artery $[16,17]$, treatment of lesions through aortocoronary grafts [18], selective contrast injection $[14,19]$ or the treatment of distal lesions in ectatic arteries [19].

Our results have highlighted the feasibility of GuideLiner use with a radial approach, achieving a high success rate when treating complex coronary lesions. In previously published series, femoral access was the most frequently used (66\% in the Twente registry [14]) and the only technique used in some reports $[13,20,21]$. In only one report, radial was the main approach [22]. The radial artery approach offers advantages over femoral access and decreases the percentage of vascular complications but provides significantly lower support [3]. This represents an important challenge when dealing with complex lesions. We are of the understanding that the transradial approach should be the first choice in most cases, and GuideLiner use may increase its effectiveness.

Radial sheathless guide catheters also enhance backup in transradial PCI, but it may still not be successful in some cases [23]. In one of the cases included in our series, we needed GuideLiner support in spite of using a Eaucath sheathless catheter (Asahi Intecc). Sheathless catheters are chosen in advance when planning complex PCI; using GuideLiner is suitable when facing unexpected delivery challenges during PCI with a regular 6-Fr guide catheter. Therefore, both techniques are complementary and can be used simultaneously.

Our data support the efficacy of this device. In all cases it was properly used, achieving a deep intubation and correct alignment in the target coronary artery. Nevertheless, in specific cases it could not be used because of significant proximal disease, marked proximal angulation or thin vessels (diameter $<2.5 \mathrm{~mm}$ ) [14, 20, 21]. Using GuideLiner resulted in optimal angiographic results in $85 \%$ of the cases where otherwise it would not have been possible to deploy a stent. These results are consistent with those from other studies, so we believe that this high efficiency makes this device a very useful tool and a first-line alternative in this kind of selected cases [14, 20-23].

It is noteworthy that GuideLiner use was feasible in acute coronary syndromes despite the thrombotic burden of that setting. In other published cohorts, GuideLiner use in acute coronary syndromes accounted for up to $40 \%$ of the total [14].

Deep coronary intubation with a guide catheter is one of the strategies that can increase support but is limited by its aggressiveness on the vessel wall [12]. Moreover, while intubating the guide, aortic wall contact is lost and the stability of the catheter decreases. The specific design of the GuideLiner minimizes arterial wall trauma and allows the guiding catheter to remain steady in the aorta while the extensor device advances in the artery. In our series, there was a proximal coronary dissection. This complication is rare, and it appears in the proximal segments of smaller arteries $[20,24]$. Another complication described is the deformation or even dislodgement of the stent before its deployment. That may occur at the transition between the hypotube and the monorail, especially if this area is located on a curve of the guide catheter $[14,25,26]$. To avoid this complication in all our cases, we placed the stent within the extensor area (monorail) while this was still in the straight section of the guide catheter (before the curve). Next, we advanced the GuideLiner and the stent (still inside the extender) simultaneously into the artery. Finally, we positioned the stent at the lesion (fig. 3). Using this strategy, we have not recorded any deterioration of the stent, and the support strength is further increased.

We did not register any of the other anecdotally reported complications, such as air embolism, deformation of the extensor or displacement of the distal marker [14, 

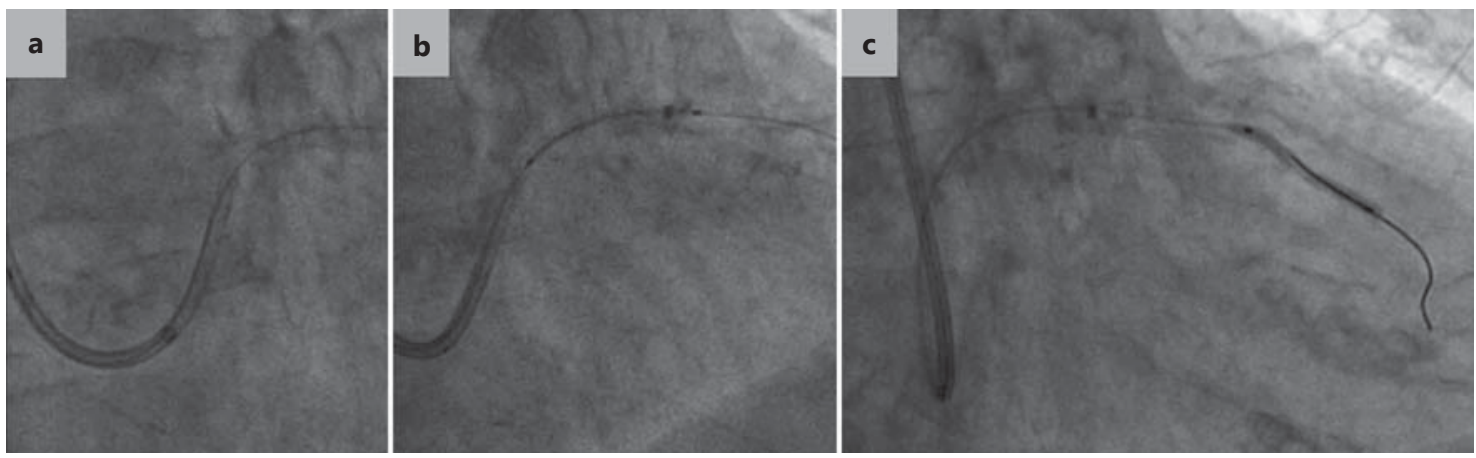

Fig. 3. 'Periscope' technique using GuideLiner. a A stent is advanced into GuideLiner while it remains in a straight section of the guide. $\mathbf{b}$ GuideLiner and the stent are advanced simultaneously into the artery. $\mathbf{c}$ The stent is further advanced out of the GuideLiner and placed in the lesion.

25]. Moreover, there were no significant clinical periprocedural events, and the reported complications could be successfully managed without further clinical implications $[14,22,24]$.

The limitations of the present study include the small number of patients from a single center, which hampered the interpretation of the results in terms of efficacy and complications. Although some alternative techniques to improve back-up and support were used, their comparison with GuideLiner efficacy lies beyond the scope of this study. Finally, device performance was not evaluated in complex bifurcations.

\section{Conclusions}

The GuideLiner extension device is an effective and safe option for the percutaneous treatment of complex lesions. Its use increased the support for advancing angioplasty balloons and stents using the radial approach, thereby improving the success rate of the procedures.

\section{Disclosure Statement}

The authors have no conflicts of interest to disclose.

\section{References}

1 Nikolsky E, Gruberg L, Pechersky L, et al: Stent deployment failure: reasons, implications and short- and long-term outcomes. Catheter Cardiovasc Interv 2003;59:324-328.

2 Hynes B, Dollard J, Murphy G, et al: Enhancing back-up support during difficult coronary stent delivery: single-center case series of experience with the Heartrail II catheter. J Invasive Cardiol 2011;23:E43-E46.

3 Hamon M, Pristipino C, Di Mario C, et al: Consensus document on the radial approach in percutaneous cardiovascular interventions: position paper by the EAPCI and Working Groups on Acute Cardiac Care and Thrombosis of the European Society of Cardiology. EuroIntervention 2013;8:1242-1251.

4 Romagnoli E, Biondi-Zoccai G, Sciahbasi A, et al: Radial versus femoral randomized investigation in ST-segment elevation acute coronary syndrome: the RIFLE-STEACS study. J Am Coll Cardiol 2012;60:2481-2489.
5 Jolly SS, Yusuf S, Cairns J, et al: Radial versus femoral access for coronary angiography and intervention in patients with acute coronary syndromes (RIVAL): a randomised, parallel group, multicentre trial. Lancet 2011;377: 1409-1420.

6 Chu G, Yang W, Zhang G, el al: Safety and efficacy of the StarClose vascular closure system following 8-Fr sheath placement for intraaortic balloon pump: a single-center analysis of 42 consecutive patients. Med Princ Pract 2014;23:313-317.

7 Joyal D, Bertrand OF, Rinfret S, et al: Metaanalysis of ten trials on the effectiveness of the radial versus the femoral approach in primary percutaneous coronary intervention. Am J Cardiol 2012;109:813-818.

8 Hamon M, Mehta S, Steg PG, et al: Impact of transradial and transfemoral coronary interventions on bleeding and net adverse clinical events in acute coronary syndromes. EuroIntervention 2011;7:91-97.
9 Saeed B, Banerjee S, Brilakis ES: Percutaneous coronary intervention in tortuous coronary arteries: associated complications and strategies to improve success. J Interv Cardiol 2008; 21:504-511.

10 Mahmood A, Banerjee S, Brilakis ES: Applications of the distal anchoring technique in coronary and peripheral interventions. J Invasive Cardiol 2011;23:291-294.

11 Burzotta F, Trani C, Mazzari MA, et al: Use of a second buddy wire during percutaneous coronary interventions: a simple solution for some challenging situations. J Invasive Cardiol 2005; 17:171-174.

12 von Sohsten R, Oz R, Marone G, McCormick DJ: Deep intubation of 6 French guiding catheters for transradial coronary interventions. J Invasive Cardiol 1998;10:198-202.

13 Kumar S, Gorog DA, Secco GG, et al: The GuideLiner 'child' catheter for percutaneous coronary intervention - early clinical experience. J Invasive Cardiol 2010;22:495-498. 
14 de Man FH, Tandjung K, Hartmann M, et al: Usefulness and safety of the GuideLiner catheter to enhance intubation and support of guide catheters: insights from the Twente GuideLiner registry. EuroIntervention 2012; 8:336-344.

15 Eddin MJ, Armstrong EJ, Javed U, et al: Transradial interventions with the GuideLiner catheter: role of proximal vessel angulation. Cardiovasc Revasc Med 2013;14:275-279.

16 Graidis C, Dimitriadis D, Karasavvidis V: Guideliner catheter facilitated percutaneous coronary artery intervention of an anteriorly displaced and heavily calcified right coronary artery. J Invasive Cardiol 2012;24:E222-E224.

17 Ramanathan K, Redfern R: Use of guideliner catheter for anomalous right coronary artery angiography and intervention. J Invasive Cardiol 2013;25:E20-E22.
18 Park C, Noble S, Bonvini RF: Guideliner microcatheter to improve back-up support during a complex coronary stenting procedure through a tortuous left internal mammary graft. J Invasive Cardiol 2012;24:E77-E79.

19 Pershad A, Sein V, Laufer N: GuideLiner catheter facilitated PCI - a novel device with multiple applications. J Invasive Cardiol 2011; 23:E254-E259.

20 Luna M, Papayannis A, Holper EM, et al: Transfemoral use of the GuideLiner catheter in complex coronary and bypass graft interventions. Catheter Cardiovasc Interv 2012;80:437-446.

21 Unzué L, Hernández F, Velázquez MT, et al: The GuideLiner catheter in complex coronary interventions. Rev Esp Cardiol 2012;65:484485.
22 Mamas MA, Fath-Ordoubadi F, Fraser DG: Distal stent delivery with GuideLiner catheter: first in man experience. Catheter Cardiovasc Interv 2010;76:102-111.

23 Harding SA, Shah N, Briggs N, Sasse A, et al: Complex transradial percutaneous coronary intervention using a sheathless guide catheter. Heart Lung Circ 2013;22:188-192.

24 Cola C, Miranda F, Vaquerizo B, et al: The Guideliner ${ }^{\mathrm{TM}}$ catheter for stent delivery in difficult cases: tips and tricks. J Interv Cardiol 2011;24:450-461.

25 Papayannis AC, Michael TT, Brilakis ES: Challenges associated with use of the GuideLiner catheter in percutaneous coronary interventions. J Invasive Cardiol 2012;24:370371.

26 Murphy JC, Spence MS: Guideliner ${ }^{\circledR}$ catheter - friend or foe? Catheter Cardiovasc Interv 2012;80:447-450. 